\title{
Substance Misuse Assessment in Mental Health Services: The Importance of Professional Curiosity
}

\section{Milani RM* \\ University of West London, UK}

*Corresponding author: Raffaella Margherita Milani, University of West London, School of Health and Human Sciences Boston Manor Rd, Brentford, UK; Email: Raffaella.Milani@uwl.ac.uk

\section{Editorial}

Co-morbidity of mental health and substance misuse problems (or "Dual Diagnosis") is highly prevalent in mental health and drug treatment services. A report commissioned by the Department of Health and NTA (National Treatment Agency) in 2002 found that 44 per cent of mental health service users either reported drug use or had used alcohol at hazardous or harmful levels in the past year [1].More recently, the 2016 National Confidential Inquiry into Suicide and Homicide by people with mental illness reported that over the last 20 years, alcohol/drug misuse and isolation have become increasingly common factors as antecedents of suicide in UK [2]. A coordinated approach between mental health and addiction services is therefore essential to prevent these clients to fall in between the gaps; however, this is far from being achieved effectively. In fact, according to the same report, more than half of the patients who died by suicide had a history of alcohol or drug misuse, but only a minority of them were in contact with substance misuse services.

The integrated approach whereby simultaneous treatment for mental and substance use disorders are offered in a single setting, has been found to be the most effective, especially when is well-managed and when the intervention is provided for a long period of time [3-6]. However, in the present UK scenario, integration of mental health and drug/alcohol services would require intervention at policy, strategic and financial level; the parallel approach, where the two conditions are treated at the same time in separate services, is the current accepted response to Dual Diagnosis. A successful parallel intervention requires well-coordinated inter-agency working, effective communication between the agencies involved and clear referral pathways [7]. Joint working and continuity of care cannot be achieved without a consistent quality drug and alcohol assessment in mental health services. The recent NICE (National Institute for Clinical Excellence) guidelines on coexisting severe mental illness and substance misuse reinforces this point by recommending that secondary mental health services should "undertake a comprehensive assessment of the person's mental health and substance misuse needs" [8].

Using the appropriate assessment tools, access to training, clear guidelines and manageable caseloads are certainly essential for implementation of routine substance misuse assessment. Ultimately, however, the qualities and skills of the individual clinician are key. Early therapeutic alliance appears to be a consistent predictor of engagement and retention in both mental health [9] and drug treatment [10]; the way that the first assessment is carried out can influence the first connection between the clinician and the service user; this important stage can be experienced as an empty "box ticking exercise" by both parties, or can become an opportunity to initiate or strengthen therapeutic engagement. Recently there has been increased attention towards the role of "professional curiosity" in the Child Protection arena [11], I would argue that this attribute plays an important role in substance misuse assessment too, especially when is carried out outside specialist drug and alcohol services. Talking about nursing education and practice, Freire (1998) defines curiosity as "The cornerstone of learning and growth...What makes me question, know, act, ask again, recognize" (p. 80) [12]. 


\section{Psychology \& Psychological Research International Journal}

There are several reasons why "professional curiosity" is important in the assessment of substance use/misuse in mental health services. One of the main aims of the assessment should be to understand the link between the client's mental health and substance use, the clinician should therefore inquiry about past and present substance use and related problems [13]. Excessive emphasis is sometimes assigned to the need of determining the order of onset of the mental and substance use disorders: "what came first?" However, these efforts may at times be driven by an attempt to try and apply a serial approach (what needs to be sorted first?) rather than working towards tackling the two issues concurrently. Trying to force a model may constitute a barrier to a client centered approach. Curiosity in this case means to be open to the unexpected and to welcome information that may not support the initial assumptions. Professional curiosity is an approach that emphasizes empathy and partnership, as such; it decreases resistance and is consistent with the spirit of Motivational Interviewing [14].

To carry out a quality substance use assessment clinicians need to be confident and comfortable asking questions about drugs, for this reason having access to training that enhances knowledge and challenges attitude towards drug users is paramount [15]. A quasiexperimental study delivered to outreach teams in Birmingham showed that was a significant improvement in knowledge and confidence in dual diagnosis interventions from pre-training to 18 months after training [16].Similarly, a randomized controlled trial study involving 79 case managers from 12 community mental health teams in South London found that dual diagnosis training successfully increased secondary measures of knowledge in Dual Diagnosis and "selfefficacy" at 18 months post-training [17].Training delivered to mental health professionals has also been shown to be effective in changing attitude towards clients with Dual Diagnosis [18].

With new psychoactive substances (NPS or former "legal highs") coming into the market every week [19], now more than ever it is easy to feel out of step [20]. In addition, most NPS users do not access traditional drug treatment services [21] and psychiatric symptoms may occur without a diagnosis of dependence or without couse of other more traditional psychoactive substances [22], in these cases mental health services become the first point of contact, consequently it is essential that frontline professionals are up to date with the new developments in the field [23]. Hence, a curious attitude is essential to nurture an independent life-long learning approach to the profession. Traditional standardised drug and alcohol screening and assessment tools often do not include NPS, it is therefore expected that they are reported under the category "other", but this option is likely to remain an empty box if the clinician has not got the necessary underpinning knowledge to prompt the right questions and the curiosity to find out more. There should be no hesitation asking clients to explain the effects of any "novel" substance and how this makes them feel. By being professionally curious the clinician therefore acknowledges the expert status of the service user when it comes to discussing their substance use.

Professional curiosity needs to be exercised also when enquiring about prescribed opioid painkillers and over the counter medications. Harry Shapiro, head of addiction charity Drug Wise, stated "We are in the midst of a great public health disaster, which is killing hundreds of people a year and ruining the lives of millions."[24] Overdose on opioid painkillers has doubled in the last decade in UK and mental health patients are amongst the high-risk groups [25].Thus, identifying use/misuse of opioid painkillers has become of critical importance in relation to assessing risks of dual diagnosis clients, especially when they are also taking CNS depressant medications.

In a multicultural society, professional curiosity means also exploring the client's treatment culture. For example, how are substance abuse and mental health problems defined by their parents, peers, other clients; what do they think should be done to remedy these problems and what kinds of treatment settings do they feel most comfortable with [26].

Professional curiosity needs to be enabled and encouraged, cannot be down solely to the good will and to the individual characteristics of the mental health professional. The British Association of Social Workers includes "Promoting a culture of professional curiosity..." as one of the Advanced Level Professional Capabilities [27].Good quality on-going training is one important aspect but organizational change also needs to be implemented in order to support front line practitioners in their role. The NMC (Nursing and Midwifery Council) recently disclosed that, in the UK, between March 2016 and May 2017, 5047 nurses left the profession outside of the retirement age because of unrealistic pressures, stress and poor management support [28]. Mental health nursing is one of the professions most at risk of "burn out" (or "compassion fatigue") [29]. Symptoms of professional burn out include: a sense of emotional and physical exhaustion, general asthenia (weakness, decreasing of activity and energy), reduced susceptibility and reaction 


\section{Psychology \& Psychological Research International Journal}

due to the changes in the external environment and a lack of curiosity response to new factors [30].Hence, the biggest obstacle to implementing routine drug and alcohol assessment in mental health services might be the assumption that this must be undertaken without any adjustment to caseload [31]. It must be recognized that meeting the multiple needs presented by clients with cooccurring disorders requires time, resources and most of all, good physical and mental health of the staff in the first place.

In conclusion, a mutual partnership between the clinician and the service user is a predictor of long term engagement. Non-judgmental professional curiosity can strengthen this alliance and reduce clients' resistance in talking about their drug use, allowing a more accurate and meaningful substance misuse assessment. By being professionally curios the clinician acknowledges the expert status of the service user and this can help strengthen the initial engagement. However, this approach needs to be enabled by a supportive and healthy working environment for mental health professionals.

\section{References}

1. Weaver T, Madden P, Charles V, Stimson G, Renton A et al. (2002) Co-morbidity of Substance Misuse and Mental Illness Collaborative Study (COSMIC) Department of Health/National Treatment Agency.

2. Appleby L, Kapur N, Shaw J, Hunt IM, Flynn S et al. (2017) The National Confidential Inquiry into Suicide and Homicide by People with Mental Illness. Making Mental Health Care Safer: Annual Report and 20-year Review. October 2016. University of Manchester.

3. Drake RE, Luciano AE, Mueser KT, Covell NH, Essock SM et al. (2016) Longitudinal Course of Clients With Co-occurring Schizophrenia-Spectrum and Substance Use Disorders in Urban Mental Health Centers: A $7-$ YearProspective Study. Schizophrenia Bulletin 42 (1): 202-211.

4. Drake RE, Mc Hugo GJ, Xie H, Fox M, Packard J et al. (2006) Ten-year recovery outcomes for clients with co-occurring schizophrenia and substance use disorders. Schizophr Bull 32(3): 464-473.

5. Wüsthoff LE, Waal H, Gråwe RW (2014) The effectiveness of integrated treatment in patients with substance use disorders co-occurring with anxiety and/or depression - a group randomized trial. BMC Psychiatry 14:67.
6. Mangrum LF, Spence RT, Lopez M (2006) Integrated versus parallel treatment of co-occurring psychiatric and substance use disorders. J Subst Abuse Treat 30(1): 79-84.

7. Schulte SJ, Meier PS, Stirling J, Berry M (2008) Treatment approaches for dual diagnosis clients in England. Drug and alcohol review 27(6): 650.

8. NICE guideline [NG58] (2016) Coexisting severe mental illness and substance misuse: community health and social care services. London: NICE.

9. Zugai JS, Stein Parbury J, Roche M (2015) Therapeutic alliance in mental health nursing: an evolutionary concept analysis. Issues Ment Health Nurs 36(4): 249257.

10. Donmall MC, Meier PS, Barrowclough C (2005) The role of the therapeutic alliance in the treatment of substance misuse: a critical review of the literature. Addiction 100(3): 304-316.

11. National Multi Agency Child Neglect Strategic Work Group (2015) Be Professionally Curious! - ction for Children. London: National Multi Agency Child Neglect Strategic Work Group.

12. Freire P (1998) Pedagogy of freedom: Ethics, democracy and civic courage. Lanham, MD: Rowman and Little.

13. Center for Substance Abuse Treatment (2005) Treatment Improvement Protocol (TIP) Series, No. 42.Substance Abuse Treatment for Persons with CoOccurring Disorders. Rockville (MD): Substance Abuse and Mental Health Services Administration (US).

14. Rollnick S, Miller WR, Butler CC (2007) Motivational Interviewing in Health Care: Helping Patients Change Behavior. NY: Guilford Press. Am J Pharm Educ 73(7): 127.

15. Moore J (2013) Dual diagnosis: training needs and attitudes of nursing staff. Mental Health Practice. 16(6): 27-31.

16. Hughes E, Wanigaratne S, Gournay K, Johnson S, Thornicroft $G$ et al. (2008) Training in dual diagnosis interventions (the COMO Study):Randomised controlled trial. BMC Psychiatry 8: 12. 


\section{Psychology \& Psychological Research International Journal}

17. Graham H, Copello A, Birchwood M, Orford J, McGovern D et al. (2006) A preliminary Evaluation of Integrated Treatment for Co-existing Substance Use and Severe Mental Health Problems: Impact on Teams and Service Users. Journal of Mental Health 15(5): 577-591.

18. Pinderup P (2017) Training Changes Professionals' Attitudes towards Dual Diagnosis. International journal of mental health and addiction 15 (1): 53-62.

19. European Monitoring Centre for Drugs and Drug Addiction (2012) Annual Report 2012 on the State of the Drugs Problem in Europe. EMCDDA.

20. Simonato P, Corazza O, Santonastaso P, Corkery J, Deluca P et al. (2013) Novel psychoactive substances as a novel challenge for health professionals: results from an Italian survey. Hum Psychopharmacol 28(4): 324-331.

21. The Royal College of Psychiatrists (2014) One new drug a week: Why novel psychoactive substances and club drugs need a different response from UK treatment providers. Lisbon: EMCDDA.

22. Stanley JL, Mogford DV, Lawrence RJ, Lawrie SM (2016) Use of novel psychoactive substances by inpatients on general adult psychiatricwards.BMJ Open 6(5).

23. Lally J (2015) Legal highs'-what's in a name. BJPsych Bull 39(4): 206.
24. New Scientist on-line (2016) Addiction to prescription drugs is UK 'public health disaster.

25. Shapiro H (2015) Opioid painkiller dependency (OPD). London: All-Party Parliamentary Group.

26. U.S. Department of Health and Human Services, Substance Abuse and Mental Health Services Administration (SAMHSA) (2005) Substance Abuse Treatment For Persons With Co-Occurring Disorders. Rockville: SAMHSA.

27. The British Association of Social Workers (2012) Advanced Level Professional Capabilities.

28. Nursing and Midwifery Council (NMC) (2017) Report on EU nurses and midwives. NMC.

29. Kasheva N, Makasheva J, Gromova A, Ishtunov S, Burykhin B (2016) The problem of professional burnout in stress management. SHS Web of Conferences 28: 01132.

30. Morse G, Salyers MP, Rollins AL, Monroe De Vita M, Pfahler C (2012) Burnout in mental health services: a review of the problem and its remediation. Adm Policy Ment Health 39(5): 341-352.

31. Padwa H, Guerrero EG, Braslow JT, Fenwick KM (2015) Barriers to serving clients with co-occurring disorders in a transformed mental health system. Psychiatr Serv 66(5): 547-550. 